\title{
Uma Medida da Percepção Pessoal da Comunicação Grupal sobre Objetos Sociais
}

\author{
João Wachelke * \\ Universidade Federal de Uberlândia, Uberlância, MG, Brasil \\ Jean Natividade \\ Pontifícia Universidade Católica do Rio de Janeiro, Rio de Janeiro, RJ, Brasil \\ Alexsandro de Andrade \\ Universidade Federal do Espírito Santo, Vitória, ES, Brasil
}

\begin{abstract}
RESUMO
O objetivo deste estudo foi construir uma medida da comunicação grupal sobre objetos sociais. Elaborou-se um instrumento e buscaram-se, para duas temáticas, evidências de validade. Participaram 803 estudantes universitários. Para ambos os temas, o instrumento mostrou-se adequado à estrutura teórica de três fatores: frequência da comunicação; importância da opinião do grupo; semelhança de opinião com o grupo. Além de apresentar índices satisfatórios de precisão, o instrumento discriminou participantes com alta e baixa identificação pessoal com o objeto social. Considera-se a medida adequada a aferir a comunicação grupal e passível de adaptação para outros grupos e objetos.
\end{abstract}

Palavras-chave: comunicação interpessoal; construção do teste; representação social.

\begin{abstract}
A Measure of the Personal Perception of In-group Communication about Social Objects

The aim of the study was to create a measure of group communication on social objects. An instrument was elaborated, and validity evidence was sought for two topics. The participants were 803 undergraduate students. For both topics, the instrument was adequate to the theoretical structure of three factors: communication frequency; importance of group opinion; similarity with group opinion. Other than presenting satisfactory indexes and precision, the instrument discriminated participants with high and low personal identification with the social object. The measure is considered suitable to assess group communication and capable of adaptation to other groups and objects.
\end{abstract}

Keywords: interpersonal communication; test construction; social representation.

Uma preocupação importante de algumas áreas da psicologia social é caracterizar crenças, pensamentos e teorias que são compartilhadas por grupos sociais. Construtos desse tipo transcendem indivíduos e dizem respeito ao conhecimento social ligado a sujeitos coletivos (Wachelke, 2012a). Um dos construtos de conhecimento social muito investigado diz respeito à noção de representação social. As representações sociais correspondem a sistemas de conhecimento, teorias do senso comum, elaborados e compartilhados por grupos, que se referem ao entendimento de como pensar e como agir na realidade social do dia a dia (Jodelet, 1989; Moscovici, 1976). Tais representações referem-se a objetos sociais, por exemplo: assuntos, questões, temas salientes e importantes para a vida dos grupos no cotidiano. Assim, para grupos com caráter político, "democracia" é um objeto social pertinente; "música" provavelmente não (Wachelke, Demantova, \& Guisso, 2012). Para estudantes, "curso universitário" é algo que os mobiliza e se qualifica como objeto social; para o grupo dos brasileiros de modo geral, "futebol" pode corresponder a um objeto social de grandes parcelas da população, e assim por diante.

Para que um objeto social constitua de fato tema de uma representação social, é pressuposto teórico a necessidade de comunicação entre os membros do grupo acerca desse objeto, pois sem comunicação não é pos- 
sível compartilhar crenças sobre esse tema, e consequentemente estruturar processos de representação social (Rouquette, 1996). Considerando-se que esse pressuposto é raramente verificado empiricamente de modo objetivo, Wachelke e colaboradores (2012) propuseram medir algumas dimensões possíveis da percepção individual de estudantes universitários a respeito da comunicação que eles mantinham com outros estudantes a respeito de alguns objetos sociais. No estudo mencionado, os autores propuseram medir três aspectos, provavelmente não exaustivos e possivelmente correlacionados entre si. Uma primeira dimensão foi chamada de frequência de comunicação sobre o objeto, e diz respeito à saliência quantitativa de um assunto em interações sociais. Nessa dimensão considera-se que é essencial que um tema seja discutido com alguma intensidade para mobilizar o debate social e a formação de posicionamentos compartilhados no grupo. À segunda dimensão foi dado o nome de importância percebida das opiniões de membros do grupo sobre o objeto. Parte do pressuposto de que opiniões grupais consideradas importantes estariam associadas a uma maior propensão de levar em conta a cultura grupal ao lidar com o assunto dessa opinião; em contraste, quando não se dá importância à opinião do grupo, pode-se lidar tranquilamente com o tema dentro de uma esfera pessoal com menos influência normativa do grupo. A última dimensão refere-se à concordância estimada com as opiniões do grupo sobre o objeto, e assim liga-se ao consenso percebido no grupo acerca de como pensar o objeto. Uma representação social tem algum consenso como condição de existência.

Devido ao caráter exploratório e propositivo dessas dimensões, elas foram mensuradas por Wachelke e colaboradores (2012) com itens únicos com respostas ordinais tratadas como numéricas posteriormente. Ainda assim, foi possível realizar uma pesquisa com 120 estudantes universitários que completaram avaliações a respeito dessas dimensões para doze possíveis objetos sociais pertinentes para o grupo dos estudantes (curso universitário, namoro, amizade, etc.), de modo que foi possível avaliar quais deles se enquadravam melhor às exigências teóricas de objeto social para o estudo de representações sociais.

Tendo em vista a utilidade de mensurar com adequadas evidências de validade e precisão a relação individual percebida de comunicação acerca de objetos sociais, tanto para o estudo do conhecimento soci- al como para outros possíveis interesses de pesquisa que se articulem com processos comunicacionais no intragrupo (ou grupo próprio), o presente estudo teve por objetivo construir e buscar evidências de validade e precisão de uma escala breve no formato de Likert para caracterizar as dimensões propostas de percepção da comunicação com o grupo acerca de objetos sociais.

\section{MÉTODO}

\section{Participantes}

No total, 803 estudantes universitários de oito estados brasileiros (Bahia, Espírito Santo, Mato Grosso do Sul, Pernambuco, Rio Grande do Norte, Rio Grande do Sul, Rondônia e Santa Catarina) participaram da pesquisa. O estado com mais participantes foi o Espírito Santo $(N=200,24,9 \%)$, enquanto Bahia teve o menor número $(N=39,4,9 \%)$. A maioria da amostra foi composta por mulheres $(N=527,65,6 \%)$. A média de idade foi de 22,7 anos $(D P=5,9)$. Os participantes estavam matriculados em diversos cursos de graduação em universidades públicas e privadas, abarcando as áreas da saúde, humanas, exatas e biológicas.

\section{Instrumento}

Buscou-se elaborar uma medida que expressasse a ideia central de cada uma das dimensões propostas de percepção da comunicação intragrupo sobre o objeto na forma de afirmações com as quais os participantes expressariam seu grau de acordo ou desacordo. Os itens do instrumento foram estruturados com o propósito de adaptação para utilização em questionários que pudessem abordar qualquer assunto (objeto social) e qualquer grupo; para tal serão indicados com colchetes, [ ], nos trechos passíveis de substituição pelos nomes de objetos e grupos. Assim, para cada dimensão foram elaborados três itens.

Referente à dimensão de frequência percebida de comunicação com o grupo (fp) os itens elaborados foram: "Converso com os [membros do grupo] frequentemente sobre [objeto]" (fpl), "[Objeto] é um assunto pouco comentado pelos [membros do grupo]" ( fp2), "Quase não falo de [objeto] com os [membros do grupo]" ( $f p 3)$. Quanto à importância percebida da opinião do grupo sobre o objeto (io), foram construídos os itens "Levo em consideração o que os [membros do grupo] pensam sobre [objeto]" (iol), "Pouco importa, para mim, o que os [membros do grupo] pen- 
sam sobre [objeto]" (io2), e "A opinião dos [membros do grupo] sobre [objeto] é importante para mim" (io3). Por fim, quanto à semelhança percebida com a opinião do grupo sobre o objeto (sp), os itens foram "Tenho uma opinião sobre [objeto] parecida com a dos [membros do grupo]" (spl), "No que diz respeito a [objeto], discordo do que pensam os [membros do grupo]" ( $s p 2)$, e "Minha opinião sobre [objeto] tem pouco a ver com a opinião dos [membros do grupo]" (sp3).

$\mathrm{O}$ instrumento de pesquisa foi um questionário apresentado como formulário on-line, construído com auxílio da plataforma Google Docs. Duas versões do questionário foram utilizadas; em cada uma delas foi abordado um objeto social diferente: ou "curso universitário" ou "namoro". O grupo incluído nos itens foi o dos "estudantes universitários". Assim, por exemplo, o item $f p 3$ para a versão "namoro" constituiu-se em: "Quase não falo de namoro com os estudantes universitários". As opções de resposta foram no formato Likert de 7 pontos, com extremos $1=$ "Discordo fortemente" e 7 = "Concordo fortemente". A sequência de apresentação dos itens da medida sobre percepção de comunicação foi, para as duas versões: $f p 2, s p 1, i o 2, f p 1, s p 2, i o 1, f p 3, s p 3$, io3.

Além dos itens construídos para aferir a comunicação intragrupal e das perguntas sociodemográficas, cada questionário trouxe também uma escala de identificação com o grupo (Wachelke, 2012b), composta por seis itens construídos a partir da literatura psicossocial, também em formato Likert de 7 pontos e com as âncoras 1 = "Discordo fortemente" e 7 = "Concordo fortemente". A escala avalia a força de conexão do indivíduo com o grupo social, no caso os estudantes universitários. Um exemplo de item é "Os estudantes universitários são um grupo importante para mim". Trata-se de uma medida unifatorial que apresentou evidências de validade em uma amostra de universitários brasileiros $(N=1203)$, para os grupos "jovens" e "estudantes universitários" com índices alfa de Cronbach 0,90 e 0,86 , respectivamente. Esse instrumento foi incluído no estudo a fim de fornecer fontes para a busca por evidências de validade com base em variáveis externas para a escala de comunicação intergrupal aqui construída (c.f. AERA, APA, \& NCME, 2014). No nível dos pequenos grupos, um resultado clássico é a associação positiva entre nível de comunicação e coesão do grupo (Lott \& Lott, 1961). Transpondo para o estudo atual, é plausível que o maior nível de identificação individual com o grupo esteja associado a maiores níveis de frequência de comunicação percebida, semelhança de opinião e percepção de importância grupal a respeito de um objeto social.

\section{Procedimento}

Os participantes foram convidados a participar da pesquisa em contextos de salas de aula de cursos de graduação de universidades públicas e particulares dos estados mencionados. Assistentes de pesquisa treinados, após agendamento de horários com professores e coordenadores, explicaram de modo geral as características da pesquisa e obtiveram cadastros de endereços de e-mail dos estudantes interessados em participar. Um convite por e-mail com um link para o questionário on-line lhes foi enviado posteriormente, sendo que a designação a uma das duas versões do questionário ocorreu aleatoriamente. As taxas de resposta finais para os dois questionários, considerando apenas questionários completos em relação ao total de convites enviados, foi de $44,6 \%$ para o questionário "curso universitário" e 47\% para "namoro". No total, 401 participantes responderam sobre namoro e 402 sobre curso universitário.

A análise de dados foi realizada separadamente para os dois objetos sociais. Inicialmente, buscaram-se evidências de validade relacionadas à estrutura interna do instrumento, por meio de análises fatoriais exploratórias; então, calculou-se o coeficiente de consistência interna (alfa de Cronbach) a fim de verificar a fidedignidade do instrumento. Evidências de validade baseadas em relações com outras variáveis também foram buscadas. Compararam-se as médias nos escores de comunicação entre subgrupos formados com os escores mais baixos de identificação grupal ( $1^{\circ}$ quartil) e os escores mais altos ( $3^{\circ}$ quartil). As análises estatísticas foram realizadas com o programa R ( R Core Team, 2013).

\section{RESULTADOS}

A fim de verificar a estrutura do instrumento construído sobre comunicação intergrupal, realizaram-se análises fatoriais exploratórias, método de eixos principais e com rotação obliqua (oblimin). No que diz respeito ao objeto curso universitário, verificou-se adequação dos dados à análise, KMO (critério de Kaiser-Meyer-Olkin) $=0,77$. Observou-se a emergência de três fatores com autovalores maiores que 1, os 
quais explicaram $53 \%$ da variância dos dados. O primeiro fator teve autovalor de 3,54, o segundo, 1,32, e o terceiro, 1,14. Um quarto fator teve autovalor 0,72 , inferior ao autovalor aleatório de 1,04 da análise paralela de autovalores aleatórios (9 itens, 402 casos, 1000 amostras bootstrap; Enzmann, 1997; Horn, 1965). Procedeu-se a mesma análise fatorial exploratória para o objeto namoro. Inicialmente, verificou-se adequação dos dados à análise, o KMO foi de 0,74 . Também para esse objeto observou-se a emergência de três fatores com autovalores maiores que 1, que explicaram $51 \%$ da variância dos dados. O primeiro fator teve autova- lor 3,45 , o segundo, 1,26 , e o terceiro, 1,18 . Um quarto fator teve autovalor 0,87 , inferior ao autovalor aleatório (9 itens, 402 casos, 1000 amostras bootstrap) da análise paralela de autovalores aleatórios, que teve valor 1,04. Assim, considerando-se os resultados encontrados e a teoria sobre o construto, sugere-se ser adequada a extração de três fatores para o instrumento.

A Tabela 1 indica as cargas fatoriais e comunalidades dos itens formulados para comunicação com o grupo sobre cada objeto.

Tabela 1

Cargas Fatoriais e Comunalidades dos Itens da Medida de Percepção de Comunicação com o Grupo sobre os Dois Objetos Sociais (Análise Fatorial Exploratória com Rotação Oblimin)

\begin{tabular}{|c|c|c|c|c|c|c|c|c|}
\hline \multirow[b]{2}{*}{ Item } & \multicolumn{4}{|c|}{ Curso universitário } & \multicolumn{4}{|c|}{ Namoro } \\
\hline & F1 & F2 & F3 & $h^{2}$ & F1 & F2 & F3 & $h^{2}$ \\
\hline$f p 1$ & 0,89 & $-0,01$ & $-0,06$ & 0,77 & 0,82 & 0,02 & 0,02 & 0,67 \\
\hline fp3 & $-0,72$ & 0,07 & $-0,05$ & 0,59 & $-0,84$ & $-0,02$ & $-0,04$ & 0,73 \\
\hline fp2 & $-0,44$ & $-0,15$ & $-0,28$ & 0,27 & $-0,35$ & 0,05 & 0,12 & 0,11 \\
\hline io3 & 0,02 & 0,94 & 0,04 & 0,85 & 0,05 & 0,96 & 0,00 & 0,89 \\
\hline io1 & $-0,08$ & 0,65 & $-0,12$ & 0,56 & $-0,12$ & 0,63 & $-0,01$ & 0,47 \\
\hline io2 & 0,07 & $-0,40$ & 0,23 & 0,32 & 0,17 & $-0,39$ & 0,17 & 0,34 \\
\hline$s p 2$ & 0,09 & $-0,03$ & $-0,74$ & 0,51 & 0,10 & $-0,03$ & $-0,74$ & 0,48 \\
\hline$s p 3$ & $-0,04$ & $-0,09$ & $-0,70$ & 0,56 & $-0,11$ & 0,06 & $-0,64$ & 0,53 \\
\hline sp1 & 0,19 & $-0,06$ & 0,44 & 0,32 & 0,08 & $-0,02$ & 0,58 & 0,40 \\
\hline
\end{tabular}

Nota. F1: fator frequência percebida $(f p)$ de comunicação com o grupo sobre o objeto; F2: fator importância percebida da opinião (io) do grupo sobre o objeto; F3: semelhança percebida de opinião com o grupo sobre o objeto. Curso universitário, $N=402$; Namoro, $N=401$.

Observa-se que a estrutura e padrão de cargas dos objetos curso superior e namoro é virtualmente idêntica, correspondendo às três dimensões previstas na construção do instrumento: frequência percebida de comunicação com o grupo sobre o objeto (Fator 1), importância percebida da opinião do grupo sobre o objeto (Fator 2) e semelhança percebida de opinião com o grupo sobre o objeto (Fator 3 ).

Quanto à confiabilidade, para o objeto curso universitário, o alfa de Cronbach foi de 0,72 para frequência de comunicação, 0,76 para importância da opinião do grupo, e 0,69 para semelhança percebida de opinião com o grupo. Para o objeto namoro, o fator frequência teve alfa 0,68 , o fator importância teve 0,75 e o fator de semelhança teve 0,72 . Assim, todas as subescalas tiveram índices satisfatórios, mesmo com pequeno número de itens em cada uma. Para os cálculos dos indicadores de confiabilidade e de validade de critério, os itens com cargas negativas tiveram seus escores invertidos.

A partir dos cálculos dos escores de cada fator, foram avaliadas as correlações entre as dimensões do instrumento, observando-se valores moderados. Para os dois objetos, houve correlações positivas e muito parecidas em termos de magnitude: entre as dimensões de frequência de comunicação e importância de opinião, $r_{\text {Curso }}=0,43 ; r_{\text {Namoro }}=0,38$, da semelhança de opinião grupal com importância da opinião do grupo, $r_{\text {Curso }}=0,43 ; r_{\text {Namoro }}=0,42$, e da semelhança de opinião com frequência percebida de opinião, $r_{\text {Curso }}=0,36$; $r_{\text {Namoro }}=0,35$, todas ao nível de $p<0,001$.

Finalmente, foram realizados testes $t$ de amostras independentes comparando os escores médios dos participantes nas três dimensões de percepção da co- 
municação com o grupo, considerando-se como grupos de comparação os participantes situados até o primeiro quartil de escores de identificação com o grupo (grupo de menor identificação - baixa id.) e o grupo situado do terceiro quartil até o valor máximo (grupo de maior identificação - alta id.), ou seja, os grupos foram classificados conforme nível de identificação dos participantes, e posteriormente compararam-se as médias obtidas na medida aqui desenvolvida, isto é, nas três dimensões de comunicação grupal sobre os objetos considerados. Para os participantes que responderam o questionário sobre curso superior, os valores do primeiro e terceiro quartil na identificação com o grupo foram 5,67 e 7,00, definindo um grupo de menor identificação com 104 participantes e um com maior identificação com 107. Quanto ao questionário de namoro, os quartis foram 5,30 e 6,70, e o grupo com menor identificação teve 93 participantes, e o de maior teve 94 .

Os resultados sinalizam diferenças nas direções esperadas, para a frequência percebida de comunicação, a média dos participantes com alta identificação é maior que os de menor identificação tanto para o objeto curso universitário, $t(208,6)=7,77 ; p<0,001 ; d=$ $1,08\left(M_{\text {alta id. }}=6,18 ; D P=1,21, M_{\text {baixa id. }}=4,87 ; D P=\right.$ $1,23)$, quanto para namoro, $t(175,9)=4,76 ; p<0,001$; $d=0,72\left(M_{\text {alta id. }}=5,12 ; D P=1,57, M_{\text {baixa id. }}=4,13\right.$; $D P=1,26)$. Isso ocorre também com a importância da opinião do grupo para os objetos curso universitário, $t(206,8)=9,83 ; p<0,001 ; d=1,37\left(M_{\text {alta id. }}=5,93\right.$; $\left.D P=1,13, M_{\text {baixa id. }}=4,34 ; D P=1,22\right)$, e namoro, $t(168,2)=5,58 ; p<0,001 ; d=0,86\left(M_{\text {alta id. }}=4,39\right.$; $\left.D P=1,64, M_{\text {baixa id. }}=3,21 ; D P=1,20\right)$. E para a dimensão de semelhança de opinião para os objetos curso, $t(205,9)=7,1 ; p<0,001 ; d=0,99\left(M_{\text {alta id. }}=\right.$ 5,$\left.56 ; D P=1,19, M_{\text {baixa id. }}=4,47 ; D P=1,02\right)$, e namoro, $t(166,4)=5,21 ; p<0,001 ; d=0,81\left(M_{\text {alta id. }}=4,59\right.$; $\left.D P=1,45, M_{\text {baixa id. }}=3,63, D P=1,04\right)$.

\section{DISCUSSÃO}

O estudo teve por objetivo elaborar e buscar evidências de validade de uma medida de percepção da comunicação com membros do grupo acerca de objetos sociais (curso universitário e namoro), inspirado na proposta de Wachelke e colaboradores (2012) que introduziram essas dimensões conceitualmente. A medida elaborada consistiu em uma escala breve, no formato de Likert, com três itens para cada dimensão: frequência percebida de comunicação com o grupo sobre o objeto, importância percebida da opinião do grupo sobre o objeto e semelhança percebida da opinião do grupo sobre o objeto. Os resultados sugerem adequadas evidências de validade e fidedignidade para o instrumento. Os padrões de cargas fatoriais indicam baixa sobreposição de cargas em fatores diferentes, e os índices de consistência interna são satisfatórios (c.f. Nunnally, 1978), especialmente considerando-se a baixa quantidade de itens do instrumento. As diferenças de escores associadas a maior ou menor identificação com o grupo são coerentes com a expectativa de maior nível de comunicação associada a um grupo mais coeso (cf. Lott \& Lott, 1961) e reforçam as evidências de validade do instrumento.

Há algumas contribuições identificadas para a utilização dessa escala. A mais importante, certamente, é a possibilidade de medir algumas propriedades psicossociais com mais precisão e a segurança de avaliar um construto e suas dimensões, em comparação com itens únicos ou outras formas de avaliação. Assim, considerando-se a importância de processos comunicacionais na interface entre indivíduo e grupo na psicologia social (Abric, 1999), trata-se de um recurso metodológico a mais disponível para pesquisadores da área.

Um segundo ponto positivo do instrumento é seu tamanho, razoavelmente breve com apenas nove itens. Questionários breves reduzem a percepção de custo cognitivo da atividade por parte do respondente (Sharp \& Frankel, 1983). Questionários longos, além de custosos para serem respondidos, podem reduzir a qualidade das respostas (Herzog \& Bachman, 1981). Por fim, um terceiro benefício diz respeito à possibilidade de adaptar a medida para grupos e objetos variados, procedimento que permite medidas com maior capacidade de generalização (p. ex. Wachelke, 2012b).

A escala proposta pode ser útil em estudos para aferir relações de integração entre fenômenos individuais e coletivos na psicologia social. Se no contexto de construtos como as representações sociais, crenças compartilhadas e atitudes sociais é pertinente fazer referência a propriedades emergentes de caráter cognitivo e afetivo por parte de grupos, torna-se importante ser capaz de avaliar, no nível dos indivíduos, se de fato eles se sentem ligados aos grupos considerados, se compartilham informações e trocas a respeito de assuntos do interesse do grupo, e assim por diante. 
Medidas de identificação com o grupo (como, por exemplo, a utilizada nesta pesquisa e apresentada em Wachelke, 2012b) cumprem a função de medir o primeiro desses aspectos. A escala apresentada neste texto possibilita a mensuração de percepções individuais sobre a comunicação com o grupo acerca de temáticas relativas a este. Pode ser incluída em projetos de pesquisa de base, permitindo assim avaliar, dentre outras possibilidades de pesquisa, a influência na estruturação de esquemas cognitivos e afetivos individuais desses padrões de comunicação, quanto identificar a formação de padrões grupais.

Quanto a limitações do estudo e da medida, é essencial apontar que não se trata de um instrumento exaustivo das dimensões de percepção de comunicação com o grupo, pois não foi feito um levantamento com essa característica previamente à construção dos itens. Neste ponto, cabe a ressalva de que apesar de vantajosos, instrumentos reduzidos podem apresentar limitações metodológicas (e.g., Natividade \& Hutz, 2015) e destaca-se a importância de continuidade do desenvolvimento de instrumentos sobre essa temática. Além disso, reconhece-se a constante necessidade por buscas de novas evidências de validade e possíveis refinamentos para a medida, a fim de melhor compreender suas potencialidades e problemas. É necessário também fazer um alerta: a medida é adaptável, em princípio, para uso junto a quaisquer objetos sociais e grupos de referência; contudo, nada indica que as evidências de validade e precisão relatadas neste trabalho reproduzam-se universalmente. $\mathrm{O}$ estudo restringiu-se aos objetos curso universitário e namoro, e junto a grupos de estudantes. Caberá aos pesquisadores que adaptarem a medida a outros objetos e grupos verificar se o instrumento apresentará adequadas evidências de validade. $\mathrm{O}$ acúmulo de dados propiciado pelo uso em diversos contextos de pesquisa por parte da comunidade acadêmica permitirá refinar o conhecimento acerca das características e estabilidade da medida.

\section{REFERÊNCIAS}

Abric, J.-C. (1999). Psychologie de la communication: théories et méthodes. Paris: Armand Colin.

American Educational Research Association, American Psychological Association \& National Council on Measurement in Education (2014). Standards for educational and psychological testing. Washington, DC: American Educational Research Association Publications.

Enzmann, D. (1997). RanEigen: A program to determine the parallel analysis criterion for the number of principal components. Applied Psychological Measurement, 21, 232.

Herzog, A. R., \& Bachman, J. G. (1981). Effects of questionnaire length on response quality. Public Opinion Quarterly, 45, 549-559.

Horn, J. L. (1965). A rationale and test for the number of factors in factor analysis. Psychometrika, 30, 179-185.

Jodelet, D. (1989). Représentations sociales: un domaine en expansion. In D. Jodelet (Org.). Les représentations sociales (pp. 31-61). Paris: PUF.

Lott, A. J., \& Lott, B. E. (1961). Group cohesiveness, communication level and conformity. Journal of Abnormal and Social Psychology, 61, 408-412.

Moscovici, S. (1976). La psychanalyse: son image et son public. Paris: PUF.

Natividade, J. C., \& Hutz, C. S. (2015). Escala reduzida de descritores dos cinco grandes fatores de personalidade: prós e contras. Psico(PUCRS), 46(1), 79-89. DOI: 10.15448/1980-8623.2015.1.16901

Nunnally, J. C. (1978). Psychometric theory ( $2^{\text {nd }}$ ed.). New York: McGraw Hill.

Pasquali, L. (2003). Psicometria: teoria dos testes na psicologia e educação. Petrópolis: Vozes.

R Core Team (2013). R: a language and environment for statistical computing. Vienna: R Foundation for Statistical Computing.

Rouquette, M.-L. (1996). La communication sociale. Paris: Dunod.

Sharp, L. M., \& Frankel, J. (1983). Respondent burden: a test of some common assumptions. Public Opinion Quarterly, 47, 36-53.

Wachelke, J. (2012a). Representations and social knowledge: an integrative effort through a normative structural perspective. New Ideas in Psychology, 30, 259-269.

Wachelke, J. (2012b). Identificação com o grupo: adaptação e validação de uma medida geral para o contexto brasileiro. Psicologia e Saber Social, 1, 187-200.

Wachelke, J., Demantova, A., \& Guisso, L. (2012). An exploratory study of the perception of ingroup communication relative to social representation objects. Psychology of Language and Communication, 16, 201-211.

Recebido em: 20/05/2013 Última revisão em: 06/05/2015 Aceito em: 20/08/2015 


\section{ANEXO 1 \\ ESCALA DE COMUNICAÇÃO INTRAGRUPAL SOBRE OBJETOS SOCIAIS}

\section{Objeto: namoro; Grupo: estudantes universitários.}

Leia as afirmações abaixo e assinale ao lado a alternativa que mais se aproxima do quanto você concorda com elas. Observe que os valores variam de 1 - Discordo fortemente, até 7 - Concordo fortemente. Quanto mais próxima das extremidades for a sua resposta, mais fortemente você discorda ou concorda. Respostas perto das posições centrais indicam que você tem uma opinião menos forte, mais indecisa.

\begin{tabular}{|c|c|c|c|c|c|c|c|}
\hline & $\begin{array}{c}\text { Discordo } \\
\text { fortemente } \\
1\end{array}$ & 2 & 3 & 4 & 5 & 6 & $\begin{array}{c}\text { Concordo } \\
\text { fortemente } \\
7\end{array}$ \\
\hline $\begin{array}{l}\text { - Namoro é um assunto pouco comentado pelos estudantes } \\
\text { universitários }\end{array}$ & 1 & 2 & 3 & 4 & 5 & 6 & 7 \\
\hline $\begin{array}{l}\text { - Tenho uma opinião sobre namoro parecida com a dos } \\
\text { estudantes universitários }\end{array}$ & 1 & 2 & 3 & 4 & 5 & 6 & 7 \\
\hline $\begin{array}{l}\text { Pouco importa, para mim, o que os estudantes } \\
\text { universitários pensam sobre namoro }\end{array}$ & 1 & 2 & 3 & 4 & 5 & 6 & 7 \\
\hline $\begin{array}{l}\text { - Converso com os estudantes universitários frequentemente } \\
\text { sobre namoro }\end{array}$ & 1 & 2 & 3 & 4 & 5 & 6 & 7 \\
\hline $\begin{array}{l}\text { - No que diz respeito a namoro, discordo do que pensam os } \\
\text { estudantes universitários }\end{array}$ & 1 & 2 & 3 & 4 & 5 & 6 & 7 \\
\hline $\begin{array}{l}\text { - Levo em consideração o que os estudantes universitários } \\
\text { pensam sobre namoro }\end{array}$ & 1 & 2 & 3 & 4 & 5 & 6 & 7 \\
\hline - Quase não falo de namoro com os estudantes universitários & 1 & 2 & 3 & 4 & 5 & 6 & 7 \\
\hline $\begin{array}{l}\text { - Minha opinião sobre namoro tem pouco a ver com a opinião } \\
\text { dos estudantes universitários }\end{array}$ & 1 & 2 & 3 & 4 & 5 & 6 & 7 \\
\hline $\begin{array}{l}\text { - A opinião dos estudantes universitários sobre namoro é } \\
\text { importante para mim }\end{array}$ & 1 & 2 & 3 & 4 & 5 & 6 & 7 \\
\hline
\end{tabular}

\section{Cálculo das Médias}

Inverter itens: $1,3,5,7,8$

Fator 1 - Frequência percebida: média aritmética dos itens 1, 4, 7.

Fator 2 - Importância percebida da opinião: média aritmética dos itens 3, 6, 9.

Fator 3 - Semelhança percebida de opinião: média aritmética dos itens 2, 5, 8 .

\section{Observação}

Os termos sublinhados representam o objeto e o grupo social, esses termos podem ser substituídos por outros objetos e grupos de acordo com o objetivo da pesquisa. No estudo de elaboração do instrumento foram encontradas evidências de validade para o grupo 'estudantes universitários' e dois objetos sociais: 'namoro' e ‘curso universitário'. 